\title{
Influence of flexion angle of files on the decentralization of oval canals during instrumentation
}

\section{Maria Antonieta Veloso Carvalho de OLIVEIRA ${ }^{(a)}$ \\ Letícia Duarte ALVES(a) \\ Analice Giovani PEREIRA(a) \\ Luís Henrique Araúio RAPOSO(b) \\ João Carlos Gabrielli BIFFI(a)}

(a) Universidade Federal de Uberlândia UFU, School of Dentistry, Department of Endodontics, Uberlândia, MG, Brazil.

(b) Universidade Federal de Uberlândia UFU, School of Dentistry, Department of Occlusion, Fixed Prosthodontics and Dental Materials, Uberlândia, MG, Brazil.

Declaration of Interests: The authors certify that they have no commercial or associative interest that represents a conflict of interest in connection with the manuscript.

Corresponding Author:

Maria Antonieta Veloso Carvalho de Oliveira E-mail: mariaoliveira@ufu.br

DOI: 10.1590/1807-3107BOR-2015.vol29.0078

Submitted: Dez 11, 2014 Accepted for publication: Feb 27, 2015 Last revision: May 15, 2015

\begin{abstract}
The aim of this study was to evaluate the influence of the flexion angle of files on the decentralization of root canals during instrumentation. Fifteen lower incisors were instrumented with Protaper Universal files and radiographed in two directions (mesiodistal and buccolingual) before and after instrumentation with a \#15 K-file in position for evaluating the flexion angle of files. The specimens were also scanned before and after instrumentation using micro-computed tomography to obtain the canal area and the distance from the center position of the file to the canal walls. Sections located $1.0 \mathrm{~mm}$ (end of the canal), $3.0 \mathrm{~mm}$ (apical third), $9.0 \mathrm{~mm}$ (middle third), and $15.0 \mathrm{~mm}$ (cervical third) from the apex were verified. After instrumentation, the flexion angles of files decreased by an average of $0.76^{\circ}$ in the buccolingual direction and $1.92^{\circ}$ in the mesiodistal direction ( $p<0.001)$; the canal area increased by an average of 0.58 , $0.37,0.23$ and $0.13 \mathrm{~mm}^{2}$ from the cervical to the end of the root canal $(p<0.001)$. Non-instrumented areas were observed on the buccal and lingual walls, and effective action of files was determined on the mesial and distal walls. The sections from the end of the canal showed canal deviation toward the lingual wall, whereas the other sections showed deviation toward the buccal wall. The flexion angles of files influence the final shape of the root canal, resulting in file decentralization along the pathway of the canal.
\end{abstract}

Keywords: Endodontics; X-Ray Microtomography; Root Canal Preparation.

\section{Introduction}

The objective of endodontic treatment is to clean and shape the root canal system, maintaining its original configuration. ${ }^{1,2,3,4}$ Appropriate canal instrumentation should provide effective canal cleansing and homogeneous dentin removal from all canal walls, delivering successful debridement without excessive wear on the root structure. ${ }^{5,6}$ The centering ability or centering ratio of the instruments is essential to obtain uniform preparation, avoiding deviation of canal walls and apical stop. ${ }^{7,8}$

Consequently, with the evolution of files, the centering ability of endodontic instruments has been continuously evaluated over the years. ${ }^{1,2,3,4,6,7,7,8,9,10,11,12,13,14,15,16,17,18,19,20,21,22,23}$ Centering ability can be influenced by the characteristics of the instruments, such as alloy composition, ${ }^{1,8,22}$ cross-section, ${ }^{6,14}$ tapering, ${ }^{2,15}$ design, ${ }^{3,22}$ tip, ${ }^{5}$ manufacturing method, ${ }^{4,22}$ 
type of movement, ${ }^{10,19}$ and rotation angle. ${ }^{23}$ The influence of the root canal anatomy, such the presence of curvatures, may also be considered. ${ }^{17}$ However, no studies have reported the influence of the flexion angles of files on the centering ability of the instrument during canal instrumentation. Files inserted into root canals may be subject to flexion in the mesiodistal (MD) and buccolingual (BL) directions owing to the presence of curvature or irregularities inside the canal system. ${ }^{24}$ This occurs because, after canal preparation, files acquire a new position as a result of the force vectors generated by flexion. ${ }^{24}$ The change in position caused by the file flexion angle during canal preparation can result in deviations along the canal walls.

Therefore, to address this issue, we used digital radiography and micro-computed tomography $(\mu-\mathrm{CT})$ to evaluate the influence of file flexion angle on the decentralization of oval root canals during instrumentation.

\section{Methodology}

Fifteen human lower incisors with single root canals (Vertucci type 1), extracted for periodontal reasons were selected (following informed consent approved by the Committee for Ethics in Research of the Universidade Federal de Uberlândia - no 067/11). The teeth selected had no previous endodontic treatment, intraradicular pins, fractures, or extensive damage in the crowns. Teeth were cleaned of debris and stored in saline solution until use. Coronal opening was performed using round diamond burs (KG Sorensen, Barueri, Brazil), and the root canals were located and explored with a \#10 K-file (Dentsply Maillefer, Ballaigues, Switzerland). The working length was determined by subtracting $1 \mathrm{~mm}$ from the length measured when the tip of the file was first observed emerging from the apical foramen. Radiographs were taken in two directions (MD and BL) with a \#15 K-file in the working length position before and after instrumentation. Teeth were instrumented with rotary files, Protaper Universal (Dentsply Maillefer) up to an F3 file, following the manufacturer's instructions. An X-Smart rotary motor (Dentsply Maillefer) with a $16: 1$ reduction hand piece and $2.0 \mathrm{~N} / \mathrm{cm}$ torque at $300 \mathrm{rpm}$ was utilized to passively introduce the files into the root canal. Irrigation was performed with 1 $\mathrm{mL}$ of $1 \%$ sodium hypochlorite after each instrument. Following the protocol described by Pereira et al..$^{24}$ for standardization of radiographs and assessment of the flexion angles of files, the radiographs taken before and after instrumentation were evaluated using Image Tool 3.00 software (University of Texas Health Science Center at San Antonio, San Antonio, USA), and the flexion of the files was determined as the angle formed between the divergence of the file path and its virtual image represented by the direction taken by the file in the absence of curvatures.

Specimens were also scanned before and after instrumentation using $\mu$-CT (SkyScan 1172; Bruker microCT, Kontich, Belgium) with an isotropic pixel size of $19.6 \mu \mathrm{m}, 90 \mathrm{kV}$, and $112 \mathrm{~mA}$, resulting in the acquisition of 1000 cross-sections per tooth at each stage. The total area of the root canal was measured before (Figure 1A) and after (Figure 1B) instrumentation in cross-sections located $1.0 \mathrm{~mm}$ (end of the canal), $3.0 \mathrm{~mm}$ (apical third), $9.0 \mathrm{~mm}$ (middle third), and $15.0 \mathrm{~mm}$ (cervical third) from the apex. In the post-instrumentation images, the canal areas were divided into the following areas: 1 -instrumented area outside the canal limits; 2- instrumented area inside the canal limits; and 3- non-instrumented area (Figure 2A). The centering ability of files was checked in the post-instrumentation images by measuring the vertical and horizontal lines of the distances from the center position of files to the limits of the original root canal in the BL and MD directions (Figures 2B and $2 \mathrm{C})$. For this, the original root canal area determined in the pre-instrumentation images was overlapped with the respective post-instrumentation images, allowing determining the action area of the files. All measurements were performed using CTAn V.1.11 scanning software (Bruker microCT).

Data were subjected to Shapiro-Wilk and Kolmogorov-Smirnov tests. The flexion angles of files (radiographs) and the total canal area $(\mu-\mathrm{CT})$ verified in the MD and BL directions before and after instrumentation passed the normality test and were analyzed with the paired t-test. Comparisons of the post-instrumentation measurements of canal areas in root cross-sections and of the distances from the center position of the file to the limits of the root canal $(\mu-\mathrm{CT})$ 


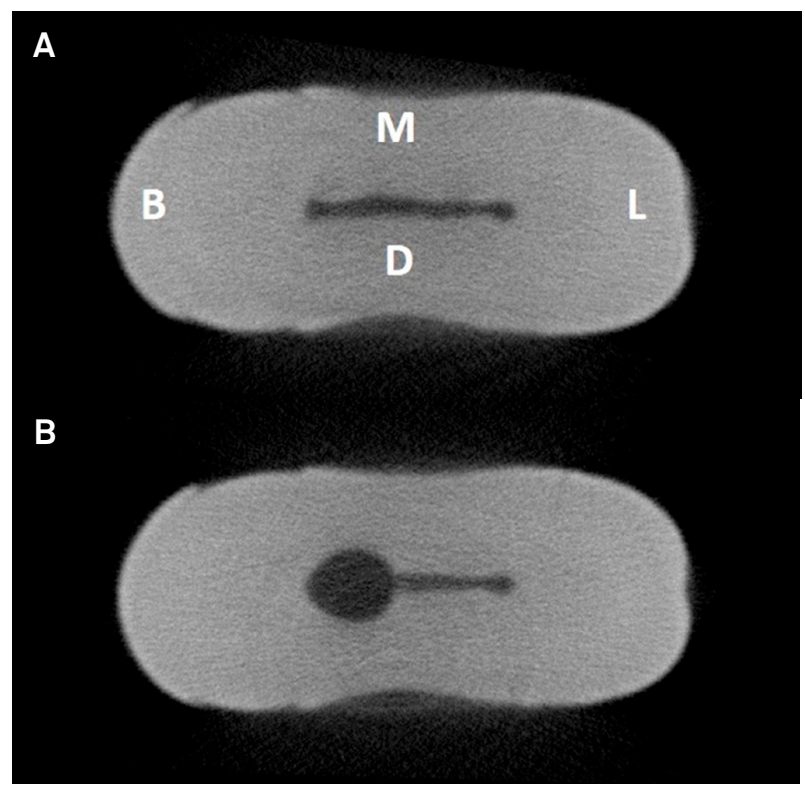

Figure 1. Root cross-sections of the middle third. A - Before instrumentation. B - After instrumentation. Root aspects: B, Buccal; L, Lingual; M, Mesial; D, Distal.

did not pass the normality test and were therefore analyzed with the Kruskall-Wallis and Dunn's tests. All tests utilized the $95 \%$ confidence interval and were performed with SigmaPlot v.12.0 statistical software (Systat Software Inc., Chicago, USA).

\section{Results}

Significant differences were verified for the flexion angles of files before and after instrumentation in the two radiographic directions $(\mathrm{p}<0.001)$. The average decrease in file angle after instrumentation was $0.76^{\circ}$ (from $0.81^{\circ}$ to $0.05^{\circ}$ ) in the BL direction and $1.92^{\circ}$ (from $2.38^{\circ}$ to $0.46^{\circ}$ ) in the MD direction.

The instrumentation significantly increased the root canal area $(p<0.001)$ in all analyzed sections. The increase was from 0.61 to $1.19 \mathrm{~mm}^{2}$ in the cervical third, from 0.35 to $0.72 \mathrm{~mm}^{2}$ in the middle third, from 0.17 to $0.40 \mathrm{~mm}^{2}$ in the apical third, and from 0.14 to $0.27 \mathrm{~mm}^{2}$ in the end of the canal. Table 1 shows the canal areas measured in the different root cross-sections after instrumentation, considering the preparation inside and outside the canal limits and non-instrumented areas. Non-instrumented areas were observed only in the buccal and lingual surfaces, with larger areas in the cervical $(p=0.042)$, middle $(p<0.001)$, and apical

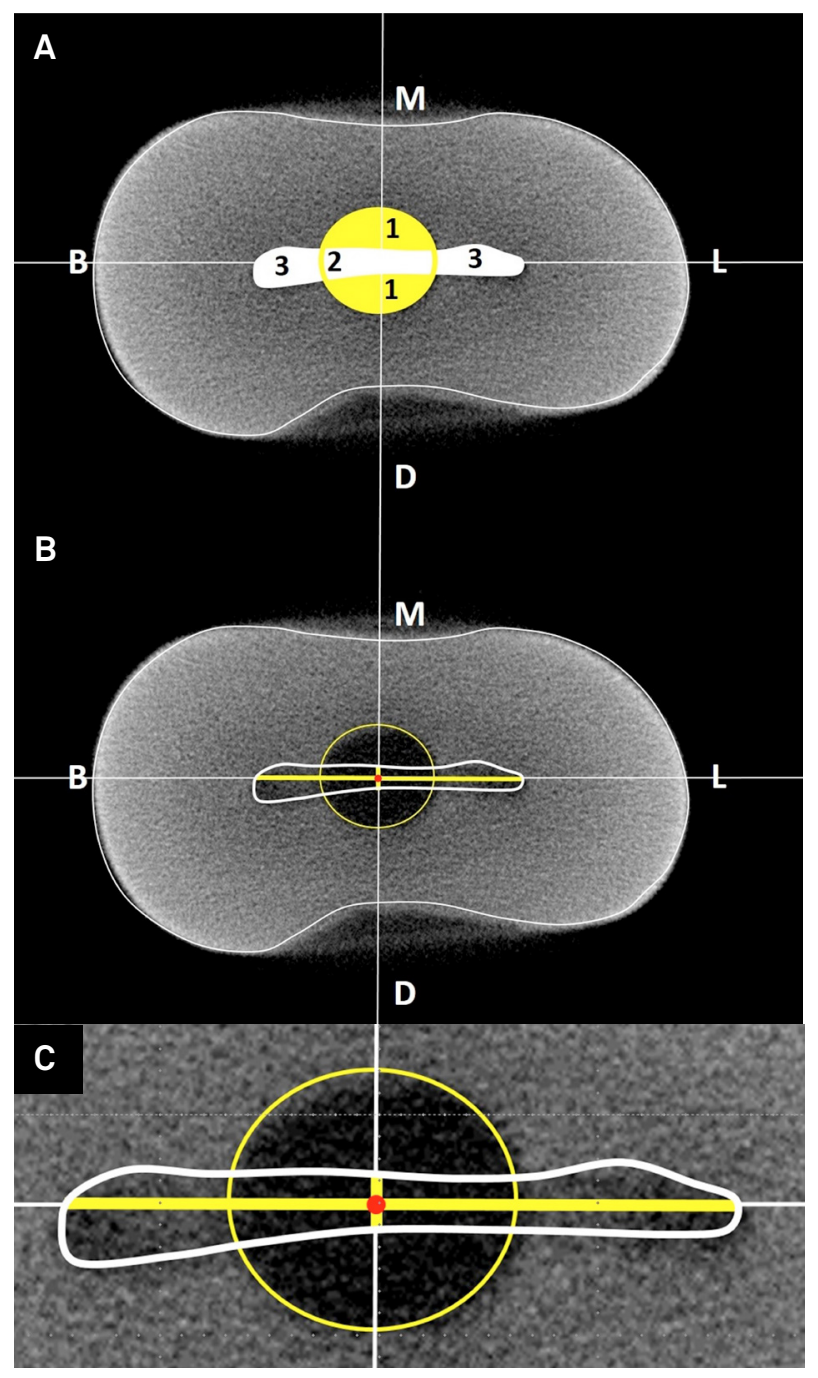

Figure 2. Post-instrumentation cross-sections of the cervical third. A - Areas of the root canal: (1) instrumentation outside canal limits, (2) instrumentation inside canal limits, and (3) non-instrumented. B - Distances (yellow lines) from the center position of files (red dot) to the limit of the original root canal (white line) in the buccolingual and mesiodistal directions. C - Magnification of the previous image showing the limits of the original root canal before instrumentation (white perimeter), the action area of files (yellow perimeter), and the buccolingual and mesiodistal lines used for the measurements (yellow lines). Root aspects: B, Buccal; L, Lingual; M, Mesial; D, Distal.

$(p=0.002)$ thirds of the buccal surface and in the lingual surface of the end of the canal $(p=0.033)$. The areas of instrumentation outside the root canal limits were concentrated in the mesial and distal surfaces, presenting no significant differences in cervical $(p=0.068)$, middle $(p=0.775)$, apical $(p=0.793)$, and end of the canal sections $(p=0.051)$. 
The mean distances from the center position of files (instrumented area) to the limits of the root canal toward each dental face were analyzed in the $\mu$-CT sections that had been used to obtain the centering ratio (Table 2). In the BL direction, the distance from the center position of the file towards the lingual region increased for the cervical, middle, and apical thirds, whereas for the end of the canal, this tendency was verified in the buccal region. In the MD direction, there were no significant differences in the distances towards the mesial and distal regions.

\section{Discussion}

For years, the centering ratio of endodontic files inside a root canal has been used as a parameter to evaluate

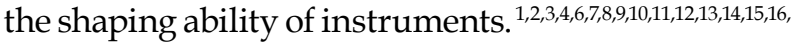
$17,18,19,20,21,22,23$ Several methods have been used to verify the centering ability of files, including longitudinal ${ }^{3,8,17}$ and transversal ${ }^{1,2,6,7,9,10,11,12,13,14,15,16,18,19,20,21,22,23}$ analysis of teeth through photographs $3,7,8,9,10,11,12,13,14,15,17,18$ and the use of cone-beam ${ }^{4,16,20,23}$ or spiral ${ }^{1,6,22}$ conventional computed tomography and $\mu-C T ., 219,21$ Most studies have used the formula recommended by Gambill et al. ${ }^{7}$ for calculating the centering ratio by measuring the root canal walls before and after instrumentation in the MD direction. $24,4,6,5,16,19,20,21,22,23$ Another formula derived by Calhoun and Montgomery ${ }^{9}$ calculates the centering ratio from measurements made inside the canal limits before and after preparation in a single root direction, MD or BL. 10,11,13,14

In the present work, the centering ratio was obtained with measurements made inside the canal limits in both the MD and BL directions of the root, taking into account the position of the file in relation to the canal limits rather than just the position of the canal. ${ }^{9,10,11,13,14,18}$ This approach allowed to determine that the centering ability of a file may vary depending on the direction of the root analyzed. Our results revealed no post-instrumentation differences in distances measured in the MD direction, implying an excellent centering ratio in all thirds. When analyzing measurements made in the BL direction, however, canal deviation was verified in $23.1 \%$ of sections from the cervical third and in $100 \%$ of sections from the middle and apical thirds and from the end of the root canal. In the cervical, middle, and apical thirds, the root canal was deviated in the buccal direction, whereas in the end of the canal a tendency for the canal to deviate in the lingual direction was observed. The deviation from the original canal can lead to inappropriate dentin removal, with the formation of a ledge and an elbow in the dentin wall, resulting in over preparation that weakens the tooth structures. ${ }^{5}$

Table 1. Median values $\left(\mathrm{mm}^{2}\right)$ for post-instrumentation measurements of canal areas in root cross-sections

\begin{tabular}{|c|c|c|c|c|c|}
\hline Root canal area & Cervical third & Middle third & Apical third & End of canal & p-value \\
\hline Instrumentation inside canal limits & $0.40^{\mathrm{Aab}}$ & $0.16^{\mathrm{ABb}}$ & $0.11^{\mathrm{BCab}}$ & $0.02^{\mathrm{Cb}}$ & $<0.001$ \\
\hline Instrumentation outside canal limits & $0.51^{\mathrm{Aa}}$ & $0.37^{A B a}$ & $0.20^{\mathrm{BCa}}$ & $0.13^{\mathrm{Ca}}$ & 0.003 \\
\hline Non-instrumented & $0.20^{\mathrm{Ab}}$ & $0.19^{\mathrm{Ab}}$ & $0.04^{\mathrm{Bb}}$ & $0.10^{\mathrm{ABab}}$ & $<0.001$ \\
\hline $\mathrm{p}$-value & $<0.001$ & $<0.001$ & $<0.001$ & 0.007 & \\
\hline
\end{tabular}

Capital letters indicate significant differences in the horizontal; lowercase letters indicate significant differences in the vertical; Dunn's test ( $p<0.05$ ).

Table 2. Median values of the distance $(\mathrm{mm})$ from the center position of the file to the limit of the root canal verified in root cross-sections

\begin{tabular}{|c|c|c|c|c|c|}
\hline \multirow{2}{*}{ Tooth face } & \multicolumn{4}{|c|}{ Analyzed section } & \multirow[b]{2}{*}{$\mathrm{p}$-value } \\
\hline & Cervical third & Middle third & Apical third & End of canal & \\
\hline Buccal & $1.05^{\mathrm{Aa}}$ & $0.49^{\mathrm{Ab}}$ & $0.20^{\mathrm{Bb}}$ & $0.44^{\mathrm{Ca}}$ & $<0.001$ \\
\hline Lingual & $1.07^{\mathrm{ABa}}$ & $1.34^{\mathrm{Aa}}$ & $0.55^{\text {Ba }}$ & $0.00^{\mathrm{cb}}$ & $<0.001$ \\
\hline Mesial & $0.28^{\mathrm{Ab}}$ & $0.21^{\mathrm{Ab}}$ & $0.19^{\mathrm{Ab}}$ & $0.13^{\mathrm{Ab}}$ & 0,091 \\
\hline Distal & $0.27^{\mathrm{Ab}}$ & $0.15^{\mathrm{Bb}}$ & $0.14^{\mathrm{Bb}}$ & $0.17^{\mathrm{Bb}}$ & 0.003 \\
\hline p-value & $<0.001$ & $<0.001$ & $<0.001$ & $<0.001$ & \\
\hline
\end{tabular}

Capital letters indicate statistical differences in the horizontal; lowercase letters indicate differences in the vertical; Dunn's test $(p<0.05)$. 
The reduction in the flexion angles of files during instrumentation influenced the centering ratio of files and, consequently, the instrumented area of root canals. When the file was introduced in the root, canal irregularities created force vectors in the file, propelling it in the opposite direction and causing flaring that may be out of the operator's control. ${ }^{1,24}$ Each force vector makes the file touch the canal walls in different ways and with different intensities, interfering with the centering ratio of the file and hindering proper cleaning. Moreover, this interference can also promote canal transportation, causing deformation of the apical stop, especially in the BL direction.

When our specimens were compared before and after instrumentation, the non-instrumented area inside the canal limits corresponded to $19.3 \%, 26 \%$, $17.1 \%$, and $48.6 \%$ of the total area in the cervical, middle, and apical thirds and in the end of the canal, respectively. Dentin flaring on the canal walls in the MD direction of the root occurred progressively from the sections of the cervical third to the end of the canal. The areas of flaring were equivalent to $15.4 \%$ of the cervical, $38.5 \%$ of the middle, and $92.3 \%$ of the apical thirds, respectively, and to $100 \%$ of the end of the canal section. Additionally, canal enlargement was not homogeneous in both directions of the root. While in the MD direction, canal enlargement was almost twofold compared with the original size, whereas in the BL direction most of the canal was not instrumented. These aspects can influence the success of endodontic treatment because bacterial film remains organized at these untouched walls

\section{References}

1. Gergi R, Rjeily JA, Sader J, Naaman A. Comparison of canal transportation and centering ability of twisted files, PathfileProTaper system, and stainless steel hand K-files by using computed tomography. J Endod. 2010 May;36(5):904-7.

2. Yamamura B, Cox TCC, Heddaya B, Flake NM, Johnson JD, Paranjpe A. Comparing canal transportation and centering ability of EndoSequence and Vortex rotary files by using Micro-Computed Tomography. J Endod. 2012 Aug;38(8):1121-5. when they are not completely reached by endodontic instruments and irrigating solutions..$^{25}$

The main purpose of this study was not to compare which instrumentation technique results in lower variation on the flexion angle of files or better centering ability, but rather to investigate the flexion angle and centering ability of files in the MD and BL directions of the root canal. Our results provide valuable information regarding the influence of the flexion angle of files on root canal decentralization during instrumentation. Future studies should be undertaken to compare the ability of different instrumentation techniques in both directions of the root canal.

\section{Conclusion}

According to the results of the present in vitro study, it was concluded that the flexion angles of files influence the final shape of an oval canal, resulting in file decentralization along the root canal. Future work to evaluate the centering ability of files should assess not only the MD but also the BL direction of the roots, which had the highest levels of file decentralization seen in our study.

\section{Acknowledgments}

The authors are indebted to Dr. Karla Vasconcelos and Dr. Frab Bóscolo from the Faculdade de Odontologia de Piracicaba, Universidade Estadual de Campinas (FOPUNICAMP) for the support with the micro-computed images and to the Fundação de Amparo à Pesquisa do Estado de Minas Gerais (FAPEMIG) for the financial support of the project.
3. Lim YJ, Park SJ, Kim HC, Min KS. Comparison of the centering ability of WaveOne and Reciproc nickel-titanium instruments in simulated curved canals. Restor Dent Endod. 2013 Feb;38(1):21-5.

4. Arora A, Taneja S, Kumar M. Comparative evaluation of shaping ability of different rotary NiTi instruments in curved canals using CBCT. J Conserv Dent. 2014 Jan-Feb;17(1):35-9. 
5. Kandaswamy D, Venkateshbabu N, Porkodi I, Pradeep G. Canal-centering ability: An endodontic challenge. J Conserv Dent. 2009 Jan-Mar;12(1):3-9.

6. Nagaraja S, Sreenivasa Murthy BV. CT evaluation of canal preparation using rotary and hand NI-TI instruments: An in vitro study. J Conserv Dent. 2010 Jan-Mar;13(1):16-22.

7. Gambill JM, Alder M, del Rio CE. Comparison of nickel-titanium and stainless steel hand-file instrumentation using computed tomography. J Endod. 1996 Jul;22(7):369-75.

8. Thompson M, Sidow SJ, Lindsey K, Chuang A, McPherson JC. Evaluation of a new filing system's ability to maintain canal morphology. J Endod. 2014 Jun;40(6):867-70.

9. Calhoun G, Montgomery S. The effects of four instrumentation techniques on root canal shape. J Endod. 1988 Jun;14(6):273-7.

10. Kosa DA, Marshall G, Baumgartner JC. An analysis of canal centering using mechanical instrumentation techniques. J Endod. 1999 Jun;25(6):441-5.

11. Bergmans L, Van Cleynenbreugel J, Wevers M, Lambrechts P. A methodology for quantitative evaluation of root canal instrumentation using microcomputed tomography. Int Endod J. 2001 Jul;34(5):390-8.

12. Ponti TM, McDonald NJ, Kuttler S, Strassler HE, Dumsha TC. Canal-centering ability of two rotary file systems. J Endod. 2002 Apr;28(4):283-6.

13. Song YL, Bian Z, Fan B, Fan MW, Gutmann JL, Peng B. A comparison of instrument-centering ability within the root canal for three contemporary instrumentation techniques. Int Endod J. 2004 Apr;37(4):265-71

14. Al-Sudani D, Al-Shahrani S. A comparison of the canal centering cbility of ProFile, K3, and RaCe Nickel Titanium rotary systems. J Endod. 2006 Dec;32(12):1198 -201.

15. Yang GB, Zhou XD, Zheng YL, Zhang H, Shu Y, Wu HK. Shaping ability of progressive versus constant taper instruments in curved root canals of extracted teeth. Int Endod J. 2007 Sep;40(9):707-14

16. Pasternak-Júnior B, Sousa-Neto MD, Silva RG. Canal transportation and centring ability of RaCe rotary instruments. Int Endod J. 2009 Jun;42(6):499-506.
17. Goldberg M, Dahan S, Machtou P. Centering ability and influence of experience when using WaveOne single-file technique in simulated canals. Int J Dent. 2012 Oct;2012(2012):206321:1-7.

18. González Sánchez JA, Duran-Sindreu F, de Noé S, Mercadé M, Roig M. Centring ability and apical transportation after overinstrumentation with ProTaper Universal and ProFile Vortex instruments. Int Endod J. 2012 Jun;45(6):542-51.

19. Stern S, Patel S, Foschi F, Sherriff M, Mannocci F. Changes in centring and shaping ability using three nickel-titanium instrumentation techniques analysed by micro-computed tomography ( $\mu \mathrm{CT}$ ). Int Endod J. 2012 Jun;45(6):514-23.

20. Capar ID, Ertas H, Ok E, Arslan H, Ertas ET. Comparative study of different novel nickel titanium rotary systems for root canal preparation in severely curved root canals. J Endod. 2014 Jun;40(6):852-6.

21. Gergi R, Osta N, Bourbouze G, Zgheib A C, Arbab-Chirani $R$, Naaman A. Effects of three nickel titanium instrument systems on root canal geometry assessed by microcomputed tomography. Int Endod J. 2015 Feb;48(2):162-70. doi: 10.1111/iej.12296. Epub 2014 May 12

22. Kumar BS, Pattanshetty S, Prasad M, Soni S, Pattanshetty KS, Prasad S. An in-vitro evaluation of canal transportation and centering ability of two rotary Nickel Titanium systems (Twisted Files and Hyflex files) with conventional stainless steel hand K-flexofiles by using Spiral Computed Tomography. J Int Oral Health. 2013 Sep-Oct;5(5):108-15.

23. Saber SEDM, Abu El Sadat SM. Effect of altering the reciprocation range on the fatigue life and the shaping ability of WaveOne nickel-titanium instruments. J Endod. 2013 May;39(5):685-8.

24. Pereira AG, Santos RMF, Azevedo KCM, Raposo LH, Biffi JC. Assessment of influence of flexion angles of files in apical stop preparation by using manual and rotary instrumentation techniques. J Endod. 2012 Oct;38(10):1383-6.

25. Vera J, Siqueira Júnior JF, Ricucci D, Loghin S, Fernandez N, Flores B, et al. One-versus two-visit endodontic treatment of teeth with apical periodontitis: a histobacteriologic study. J Endod. 2012 Oct;38(10):1040-52. 